\title{
Ileal follicular lymphoma with atypical endoscopic findings
}

Authors

Institution
Satoshi Yamada, Yorimitsu Koshikawa, Naoki Minami, Yusuke Honzawa, Minoru Matsuura, Hiroshi Nakase

Department of Gastroenterology and Hepatology, Graduate School of Medicine, Kyoto University, Kyoto, Japan submitted 6. October 2015 accepted after revision 23. December 2015

\section{Bibliography}

Dol http://dx.doi.org/ 10.1055/s-0042-100721 Endoscopy International Open 2016; 04: E323-E325 (c) Georg Thieme Verlag KG Stuttgart $\cdot$ New York E-ISSN 2196-9736

\section{Corresponding author}

Hiroshi Nakase, MD, PhD

Department of

Gastroenterology and

Hepatology

Graduate School of Medicine

Kyoto University

54 Kawahara-cho

Shogoin

Sakyo-ku

Kyoto

606-8507

Japan

Fax: +81-75-7514303

hiropy_n@kuhp.kyoto-u.ac.jp
A 72-year-old woman presented with symptomatic anemia without abdominal symptoms. She had no history of abdominal surgery or use of non-steroidal anti-inflammatory drugs. Enhanced computed tomography of the abdomen revealed swelling of multiple intraperitoneal lymph nodes and a high density of mesenteric adipose tissue. Fluorodeoxyglucose (FDG-) positron emission tomography showed high FDG accumulation at the intraperitoneal lymph nodes. Double-balloon enteroscopy detected severe ste-

\section{Background \\ $\nabla$}

Follicular lymphoma is the most common subtype of non-Hodgkin's lymphoma (NHL) [1,2]. Gastrointestinal follicular lymphoma mainly comprises scattered small whitish nodules, mass lesions and polyps, while other signs are exceedingly rare. The recent development of enteroscopy devices, such as double-balloon enteroscopy (DBE) and capsule endoscopy, allows gastroenterologists to detect atypical lesions of gastrointestinal follicular lymphoma. Herein, we report an unusual case of gastrointestinal follicular lymphoma presenting with an annular ulcer.

\section{Case report}

$\nabla$

A 72-year-old woman presented with symptomatic anemia without abdominal symptoms. She had no history of abdominal surgery or use of non-steroidal anti-inflammatory drugs (NSAIDs). She also had no relevant family history of medical conditions. Physical examination revealed no significant findings other than anemic palpebral conjunctiva. Laboratory findings included hemoglobin $5.9 \mathrm{~g} / \mathrm{dL}$ and mean corpuscular volume of $63.0 \mathrm{fL}$. Neither esophagogastroduodenoscopy nor colonoscopy revealed abnormal findings. Enhanced computed tomography (CT) of the abdo- nosis with an annular ulcer in the lower ileum. She was diagnosed with ileal follicular lymphoma based on histologic examination and fluorescence in situ hybridization analysis of the biopsy specimen. The ileal ulcer was successfully treated by chemotherapy with rituximab and bendamustine for 1 year. We strongly recommend consideration of gastrointestinal follicular lymphoma in the differential diagnosis of annular ulcers in the small intestine.

men revealed swelling of multiple intraperitoneal lymph nodes and a high density of mesenteric adipose tissue ( $\bullet$ Fig. 1$)$. Fluorodeoxyglucose (FDG)-positron emission tomography (PET) showed high FDG accumulation at the intraperitoneal lymph nodes. DBE was performed using a retrograde approach, and severe stenosis with an annular ulcer was detected in the lower ileum (๑ Fig. 2).

The differential diagnosis of ileal annular ulcers includes intestinal tuberculosis, NSAID-related ulcers, Crohn's disease, localized ischemia of the small intestine, and lymphoma. Viral and bacterial infections, including tuberculosis, were excluded. NSAID-related ulcers were ruled out because she had no history of taking NSAIDs. Histologic examination of biopsy specimens from the ileal ulcer revealed a concentration of medium-sized atypical lymphocyte-like cells that were immunohistochemically positive for CD10, CD20, and Bcl-2 antibodies, and negative for CD3 and CD5 antibodies ( Fig. 3). Fluorescence in situ hybridization analysis showed $\mathrm{IgH}-\mathrm{Bcl} 2$ rearrangement in $67 \%$ of the ileal ulcer cells ( $\bullet$ Fig. 3 ), indicating a chromosomal transfer of $t(14 ; 18)(q 32 ; q 21)$. She was diagnosed with primary ileal follicular lymphoma, stage II2 based on the Lugano classification due to metastasis to distant abdominal lymph nodes. 

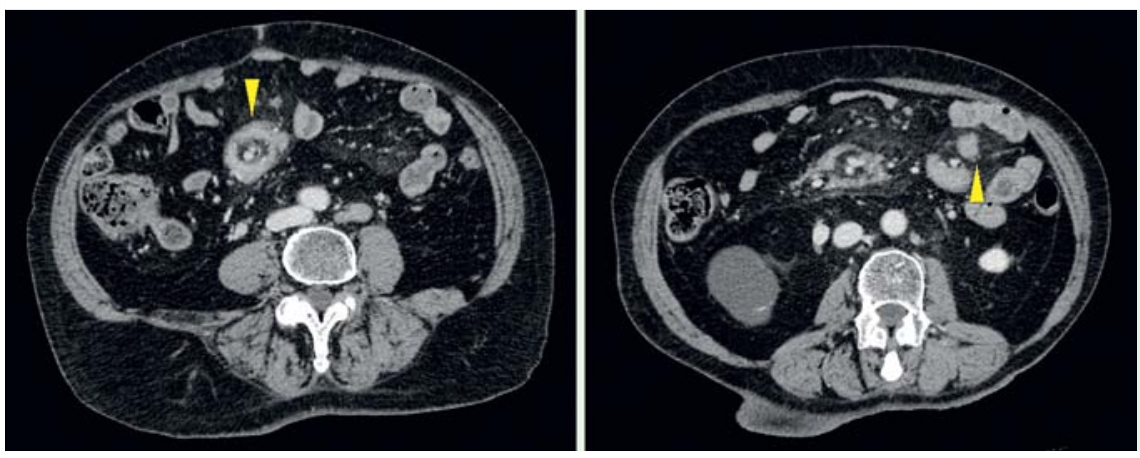

Fig. 1 Enhanced computed tomography (CT) of the abdomen revealed swelling of multiple intraperitoneal lymph nodes (arrow) and a high density of mesenteric adipose tissue.
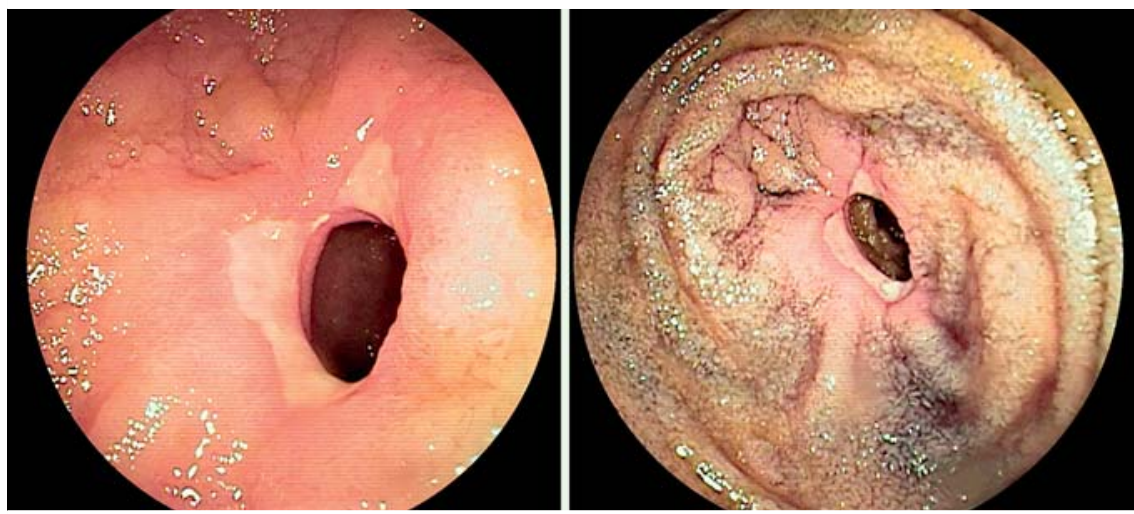

Fig. 2 Double-balloon enteroscopy (DBE) was performed using the anal approach, and severe stenosis with an annular ulcer was detected in the lower ileum.
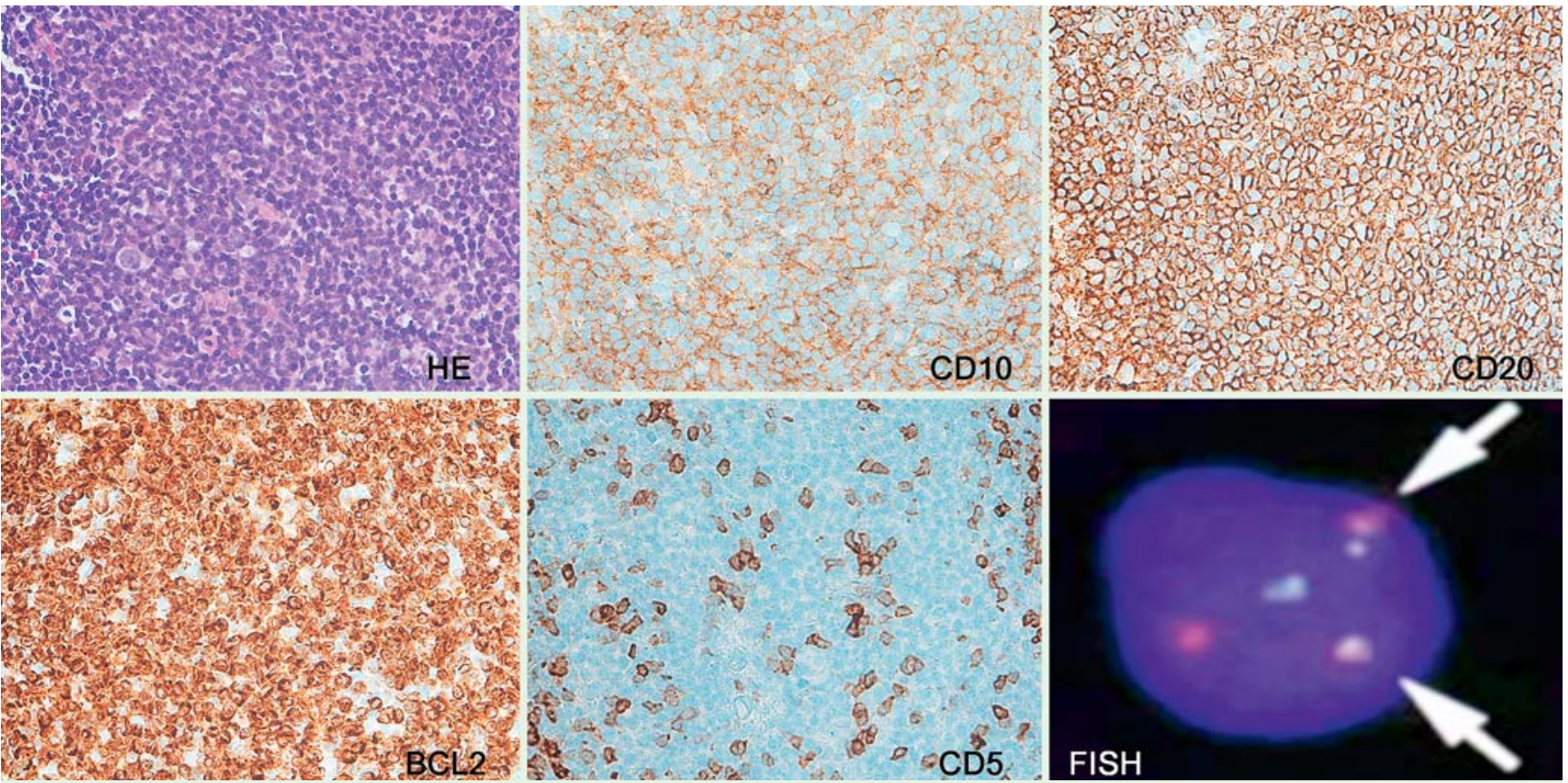

Fig. 3 Histologic examination of biopsy specimen from the ileal ulcer (photomicrograph original magnification $\times 400$ ), and fluorescence in situ hybridization (FISH) analysis.

Chemotherapy with rituximab and bendamustine was selected because of her advanced age and the low grade malignancy of follicular lymphoma. Combination chemotherapy with rituximab and bendamustine has equivalent effects and lower toxicity than polypharmacy with rituximab, cyclophosphamide, hydroxydaunorubicin, oncovin, and prednisone (R-CHOP) in NHL with low grade malignancy such as follicular lymphoma. The ileal ulcer resolved within 1 year after initiating the chemotherapy. Moreover, swelling of multiple intraperitoneal lymph nodes observed with enhanced CT, and high FDG accumulation at the intraperitoneal lymph nodes with FDG-PET also disappeared. She has had no recurrence of gastrointestinal follicular lymphoma for 3 years.

\section{Discussion}

$\nabla$

Follicular lymphoma is the most common subtype of NHL [1,2]. Most cases of follicular lymphoma are initially detected in the lymph nodes, and widespread nodal involvement such as Ann Arbor stage III or IV is a typical presentation of this disease, whereas only $5-10 \%$ of patients present with early stage I or II [3]. The 
gastrointestinal tract is the common site of extra-nodal NHL [1, 2]. Although extra-nodal involvement usually occurs as a result of disseminated nodal disease, primary gastrointestinal NHL accounts for $30-40 \%$ of all extra-nodal NHL [4]. Most common subtypes of primary gastrointestinal NHL are mucosal-associated lymphoid tissue lymphoma or high grade diffuse large B-cell lymphoma, while primary gastrointestinal follicular lymphoma is a relatively rare disease accounting for only $1 \%$ of gastrointestinal NHL [5].

Gastrointestinal follicular lymphoma lesions comprise mainly scattered small whitish nodules $1-2 \mathrm{~mm}$ in diameter and subsequent mass lesions with or without ulceration. In previous studies of 222 cases with small intestinal follicular lymphoma, scattered small whitish nodules, mass lesions, and polyps were detected as follicular lymphoma lesions in the small intestine in 183 cases ( $82.4 \%$ ), 29 cases (13.1\%), and six cases (2.7\%), respectively, and other morphology was detected in only four cases (1.8\%) [1]. Annular ulcers, such as presenting here, are exceedingly rare.

Studies performed before the introduction of DBE or capsule endoscopy revealed that gastrointestinal follicular lymphoma lesions were mostly unifocal, and the proportion of ileal localization was low [6-8]. Recent studies, however, reported that DBE detected new jejunoileal follicular lymphoma lesions in $73-$ $85.7 \%$ of cases with duodenal follicular lymphoma [6,7]. Moreover, 24 of 33 patients with gastrointestinal follicular lymphoma (72.7\%) who underwent enteroscopy had multifocal involvement, mostly in the duodenum and ileum [1]. In addition, FDGPET was not always effective in detecting gastrointestinal follicular lymphoma lesions, because three-quarters of patients with gastrointestinal follicular lymphoma who had been diagnosed by DBE were negative for FDG-PET [8]. Therefore, investigation of the small intestine is recommended for the diagnosis of gastrointestinal follicular lymphoma. With the increasing use of DBE for observation of small intestinal lesions, the frequency of gastrointestinal follicular lymphoma with atypical changes might increase.
Based on the present case, gastrointestinal follicular lymphoma should be considered in the differential diagnosis of annular ulcers in the small intestine.

Competing interests: None

\section{Acknowledgments \\ $\nabla$}

This work was supported by the Japanese Society for the Promotion of Science "KAKENHI" Grants-in-aid for Scientific Research (24590941, 25860532, and 26460967) and Health and Labour Sciences Research Grants for Research on Rare and Intractable Disease from the Ministry of Health, Labour and Welfare, Japan.

\section{References}

1 Yamamoto S, Nakase H, Yamashita $K$ et al. Gastrointestinal follicular lymphoma: review of the literature. J Gastroenterol 2010; 45: $370-$ 388

2 Damaj G, Verkarre V, Delmer A et al. Primary follicular lymphoma of the gastrointestinal tract: a study of 25 cases and a literature review. Ann Oncol 2003; 14: 623-629

3 Iwamuro M, Okada $H$, Kawano $S$ et al. A multicenter survey of enteroscopy for the diagnosis of intestinal follicular lymphoma. Oncol Lett 2015; 10: $131-136$

4 d'Amore F, Christensen BE, Brincker $\mathrm{H}$ et al. Clinicopathological features and prognostic factors in extranodal non-Hodgkin lymphoma. Danish LYFO Study Group. Eur J Cancer 1991; 27: 1201-1208

5 Morton JE, Leyland MJ, Vaughan HudsonG et al. Primary gastrointestinal non-Hodgkin's lymphoma: a review of 175 British National Lymphoma Investigation cases. Br J Cancer 1993; 67: 776 - 782

6 Kodama M, Kitadai $Y$, Shishido $T$ et al. Primary follicular lymphoma of the gastrointestinal tract: a retrospective case series. Endoscopy 2008; 40: 343-346

7 Higuchi $N$, Sumida $Y$, Nakamura $K$ et al. Impact of double-balloon endoscopy on the diagnosis of jejunoileal involvement in primary intestinal follicular lymphomas: a case series. Endoscopy 2009; 41: $175-178$

8 Ibuka T, Akari H, Sugiyama T et al. Diagnosis of the jejunoileal lymphoma by double-balloon endoscopy. World J Gastrointest Endosc 2013; 5: $111-116$ 